\title{
Artificial intelligence for predicting recurrence-free probability of non-invasive high-grade urothelial bladder cell carcinoma
}

\author{
TOMMASO CAI ${ }^{1}$, GLORIA CONTI ${ }^{2}$, GABRIELLA NESI $^{3}$, MATTEO LORENZINI $^{4}$, \\ NICOLA MONDAINI $^{1}$ and RICCARDO BARTOLETTI ${ }^{1}$ \\ Departments of ${ }^{1}$ Urology, ${ }^{2}$ Informatics Engineering, ${ }^{3}$ Pathology and Oncology, \\ and ${ }^{4}$ Physic, University of Florence, I-50011 Florence, Italy \\ Received February 20, 2007; Accepted May 3, 2007
}

\begin{abstract}
The objective of our study was to define a neural network for predicting recurrence and progression-free probability in patients affected by recurrent pTaG3 urothelial bladder cancer to use in everyday clinical practice. Among all patients who had undergone transurethral resection for bladder tumors, 143 were finally selected and enrolled. Four followups for recurrence, progression or survival were performed at 6, 9, 12 and 108 months. The data were analyzed by using the commercially available software program NeuralWorks Predict ${ }^{\circledR}$. These data were compared with univariate and multivariate analysis results. The use of Artificial Neural Networks (ANN) in recurrent pTaG3 patients showed a sensitivity of $81.67 \%$ and specificity of $95.87 \%$ in predicting recurrence-free status after transurethral resection of bladder tumor at 12 months follow-up. Statistical and ANN analyses allowed selection of the number of lesions (multiple, $\mathrm{HR}=3.31$, $\mathrm{p}=0.008)$ and the previous recurrence rate $(\geq 2 /$ year, $\mathrm{HR}=3.14$, $\mathrm{p}=0.003$ ) as the most influential variables affecting the output decision in predicting the natural history of recurrent pTaG3 urothelial bladder cancer. ANN applications also included selection of the previous adjuvant therapy. We demonstrated the feasibility and reliability of ANN applications in everyday clinical practice, reporting a good recurrence predicting performance. The study identified a single subgroup of pTaG3 patients with multiple lesions, $\geq 2$ year recurrence rate and without any response to previous Bacille Calmette-Guérin adjuvant therapy, that seem to be at high risk of recurrence.
\end{abstract}

\section{Introduction}

Urothelial carcinomas (UC) of the urinary bladder, described as non-invasive high-grade urothelial bladder cell carcinoma (pTaG3) at pathological analysis, have a frequency rate ranging

Correspondence to: Dr Tommaso Cai, Department of Urology, University of Florence, via dell'Antella 58, I-50011 Florence, Italy E-mail: ktommy@libero.it

Key words: artificial neural network, bladder cancer, superficial urothelial carcinoma, recurrence, prognosis from 2.9 to $18.0 \%$ (1-4) with recurrence and progression to muscle-invasive disease rate ranging from 65 to $85 \%(1,3,4)$ and 20 to $25 \%$ (2), respectively. No single standard prognostic factor can correctly predict the natural history of this type of bladder tumor and be used to plan a correct follow-up schedule for these patients (5). Furthermore, no evidence exists that an aggressive treatment is always useful in all patients with pTaG3 bladder cancer, particularly in terms of quality of life $(6,7)$. To increase understanding of urothelial bladder cancer biological behavior, many groups of potential molecular markers and quantitative morph metric methods have been described $(8,9)$. The lack of pathological and clinical methods to predict disease recurrence or progression has led to search for methods that combine existing clinical, pathological and laboratory data with complex mathematical models. For this reason, Artificial Neural Networks (ANNs) (10) have been introduced into clinical practice, reporting a good performance for predicting the postoperative outcome in patients with cancer (11). However, to date the ANNs have only been used rarely in urological oncological diseases (12-16). In a previous study, we demonstrated that the use of ANNs in bladder cancer outcome patient evaluation provides for valid support in follow-up and treatment schedule planning with an overall accuracy of $88.43 \%$ (17). The aim of the present study was to define a neural network for predicting recurrence and progression-free probability in patients with recurrent pTaG3 urothelial bladder cancer, developed in accordance with the results of univariate and multivariate analyses.

\section{Materials and methods}

Study design. All consecutive patients who had undergone transurethral resection of bladder tumor (TUR-BT) at the same Urologic Unit, between November 1993 and December 1996, were recruited for the study. Each patient underwent a follow-up schedule, according to the international guidelines (18). Moreover, during the TUR-BT, a bladder mucosa mapping around the tumor was also carried out, in order to evaluate the presence of associated carcinoma in situ (Cis).

Eligibility for the study. All patients who reported the presence of cystoscopically demonstrated bladder tumors were selected for the study. All patients with a history of UC 
of the upper urinary tract or with one of prostate or other urological cancers were excluded. All patients with other urological diseases were also excluded. Among all patients affected by demonstrated bladder tumors, only those who had reported a recurrent $\mathrm{pTaG} 3$ or a non-invasive tumor in progression from low-grade to high-grade (from pTaG1-G2 to pTaG3) were finally enrolled in the study. Informed consent was obtained from all patients. The study was approved by the local research ethics committee. The present study was conducted according to the ethical principles laid down in the latest version of the Declaration of Helsinki and in line with GCP (Good Clinical Practice) guidelines.

Histopathological analysis. The cases were reviewed on the basis of the available slides, in order to evaluate histotype, stage and grade of the tumor. The slides were reviewed by the same pathologist. Specimens were pathologically staged according to the 1997 TNM classification of the International Union Against Cancer and the grade was assessed following the 1973 WHO classification. Histopathological analysis included the evaluation of the tumor-associated tissue inflammatory reaction in each sample of urothelial bladder cell carcinoma as described in our previous report (19).

Clinical follow-up data collection and analysis. The mean follow-up was 108 months (ranging from 91 to 149 months). Recurrence was determined on the basis of papillary formations protruding into the bladder lumen and detected by cystoscopy or the presence of neoplastic tissue diagnosed after vesical biopsy. The recurrence rate was defined as the ratio of the number of recurrences on the total follow-up period (recurrences/years). The time to the first recurrence was defined as the time from randomization to the date of the first recurrence. Progression was defined as the increase in tumor stage. The follow-up results at 6,9 and 12 months from TUR-BT and at the end of follow-up period (mean 108 months) were recorded and used for univariate, multivariate and ANN analyses. The follow-up at 3 months after TUR-BT was recorded but not included in univariate, multivariate and ANN analyses, because an incomplete resection, implantation at traumatized sites in the bladder or rapid growth of epithelial malignancy are responsible for the higher recurrence rate of superficial UC after TUR-BT at 3 months (20), even if several authors have suggested that a recurrence at 3-month follow-up is an important prognostic factor for predicting progression in high-grade bladder tumors (21).

Statistical analysis. Pearson's coefficient was adopted to evaluate the correlation between the different parameters in all patients, and Fisher's exact test was used to assess statistical significance with $\mathrm{p}<0.05$ accepted as significant. Two-side tests of significance were performed for all analyses. Bonferroni's correction was also used. The ANOVA test was used for univariate analysis and the log-rank test (Mantel Cox) for multivariate analysis. The Cox proportional hazard regression model was also used for calculating the hazard ratio (HR) with the $95 \%$ confidence interval $(95 \% \mathrm{CI})$. Ninety-five percent confidence intervals were calculated for the probability of surviving for the Kaplan-Meier estimates. The receiver operating characteristic curve (ROC) was used to discover the best cut-off value, in the ANN analysis. The efficacy of the ANN for predicting recurrence and progressionfree probabilities in pTaG3 patients were tested with accuracy, sensitivity, specificity, positive predictive value and negative predictive value. Root Mean Square error was also used to evaluate the error between corresponding pairs of values in two series of values. Smaller Root Mean Square error values are better. The ANN methodology and data analysis will be described in detail below. All statistical analyses were performed using SPSS 11.5 for Apple-Macintosh (SPSS, Inc. Chicago, IL).

Artificial Neural Network. The impact of 9 factors on recurrence-free status at 12 months was studied. The factors finally included in the ANN analysis were: sex (male or female), age ( $<65$ or $\geq 65$ years), previous histopathological data (pTaG1, pTaG2 or pTaG3), previous recurrence rate (1 recurrence/year, 2 recurrence/year, 3 or more/year), response to previous Bacille Calmette-Guérin adjuvant therapy (yes or no), number of lesions (single or multiple), size of lesions $(1,2,3,4,5$ or more), presence of tumorassociated inflammatory reaction (yes or no) and adjuvant therapy after TUR-BT (yes or no). Two factors (associated Cis and type of previous adjuvant chemotherapy) were excluded from the ANN construction and analysis due to a limited number of patients with associated Cis or who had undergone previous adjuvant chemotherapy. The patients were defined as having response to previous Bacille Calmette-Guérin adjuvant therapy if the recurrence did not occur within 6 months of starting BCG treatment, according to Herr and Dalbagni (22). ANN setup was carried out by using the commercially available software program NeuralWorks Predict ${ }^{\circledR}$ (NeuralWare, 2005, Inc., Carnegie, PA, USA). The 9 factors were finally used as input parameters (input neuron) to ANN. All characteristics of the ANN structure are the same as described in our previous study (17). Of all selected patients, 100 (69.9\%) were used to train the system, while $43(30.1 \%)$ were used for the testing phase. After the training phase, no retraining of the network was permitted. In this study we did not perform a standard logistic regression (LR) for comparing these results to those of the ANN analysis, because the majority of authors have already demonstrated that ANN analysis gives rise to more accurate results than LR or other standard statistical analysis $(15,16)$.

\section{Results}

One hundred and forty-three consecutive recurrent pTaG3 patients were finally enrolled. Of all the enrolled patients, 116 were male $(81.1 \%)$ and 27 female (18.9\%). Patient ages ranged from 46 to 88 (mean, 67.8 years). The patient and tumor characteristics are described in Table I.

Follow-up results. At 6 and 9 months after TUR-BT, the patients alive without recurrence were $110(76.9 \%)$ and 80 $(55.9 \%)$, respectively. Fifty-five patients $(38.4 \%)$ were alive without any evidence of recurrence at mean 12 months follow-up, $86(60.1 \%)$ were alive with at least one recurrence, while two died of unrelated causes. Among all recurrent patients, only 3 reported a progression to invasive disease: 3 to pT1 (time to progression 6,7 and 11 months). Among all 
Table I. The patient and tumor characteristics and adjuvant intravesical therapy in 143 recurrent pTaG3 enrolled patients.

Patient characteristics

\begin{tabular}{lr}
\hline No. of patients & 143 \\
Sex & \\
Male & 116 \\
Female & 27
\end{tabular}

Median age (years)

$67.8 \pm 7.67$

\begin{tabular}{lc}
\hline & Clinical data \\
\hline Previous histopathological data & \\
pTaG1 & $41(28.8)$ \\
pTaG2 & $74(51.7)$ \\
pTaG3 & $28(19.5)$
\end{tabular}

No. of recurrence/year

$36(25.2)$

2

$82(57.4)$

$\geq 3$

$25(17.4)$

Previous intravesical treatment

BCG $^{\text {a }}$

$108(75.5)$

Mitomycin

None

No. of lesions

1

$56 \quad(39.1)$

2

59 (41.2)

$13(9.0)$

3

$9 \quad(6.2)$

$\geq 5$

$6 \quad(4.5)$

Diameter of lesion (if multiple,

diameter of the largest) $(\mathrm{cm})$
1-1.9
2-2.9
3-3.9
$4-4.9$
$\geq 5$

68 (47.5)

36 (25.1)

21 (14.6)

$10 \quad(6.9)$

8

Treatment plan

\section{Surgical treatment}

TUR-BT ${ }^{b}$

Adjuvant therapy

$\mathrm{BCG}^{\mathrm{a}}$

Mitomycin

Epirubicin

7

None

The table describes the clinical and histopathological data of all enrolled patients. The type of adjuvant intravesical therapy after surgery is shown. Data in parentheses are percentages. ${ }^{\mathrm{a}} \mathrm{BCG}$,

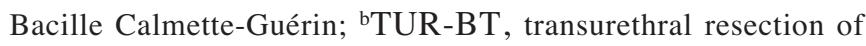
bladder tumor). enroled patients, $122(85.3 \%)$ were still alive at mean 108 months follow-up, while 21 (14.7\%) had died. Among deceased patients, 12 out of 21 died of disease progression at mean 10.6 months from TUR-BT, while 9 died owing to unrelated reasons. Overall, 120 out of $143(83.9 \%)$ patients had at least one recurrence during the follow-up period; 39 patients had only one recurrence $(32.5 \%)$, while 81 two or more recurrences $(67.5 \%)$. The mean time to first recurrence was 13 months (ranging from 5 to 31 months). The recurrence rate mean was 2 per year (ranging from 1 to 4 per year). Among the 120 patients that reported at least one recurrence, $42(35.0 \%)$ had progression to invasive disease: 35 to pT1 $(83.3 \%)$ and 7 to pT2 $(16.7 \%)$. Patients with progression to pT2 underwent radical cystectomy with urinary diversion, while those with pT1 underwent TUR-BT or radical cystectomy according to patient and tumor characteristics. The mean time to progression was 12 months (ranging from 5 to 21 months).

Univariate and multivariate analyses. Univariate analysis did not recognize any prognostic factors to use in predicting recurrence or progression probability in pTaG3 lesions. Multivariate analysis, however, selected the previous recurrence rate (number of recurrences per year) $(\mathrm{p}=0.003)$ (2 recurrence rate per year, $\mathrm{HR}=4.78,95 \% \mathrm{CI} 3.12-6.23)$ and the number of lesions ( $\mathrm{p}=0.008$ ) (multiple, $\mathrm{HR}=3.31,95 \%$ CI 2.36-3.85) as independent determinants for predicting recurrence. In addition, the presence of tumor-associated tissue inflammatory reaction $(\mathrm{p}=0.043)$ (tumor without inflammation, $\mathrm{HR}=2.89$, $95 \%$ CI 2.10-4.92) was identified as an independent prognostic factor in predicting recurrence (Table II).

Artificial Neuronal Network analysis. The use of ANN in pTaG3 patients allowed a sensitivity of $81.67 \%$ and specificity of $95.87 \%$ in predicting recurrence-free status after TUR-BT at 12 months follow-up (positive predictive value, 98.2\% and negative predictive value, $92.3 \%$ ). The area under the ROC curve was 0.816 (Fig. 1). All diagnostic parameters affecting output decision used for construction of ANN are shown in Table III. ANN analysis reported a high correlation between expectant and observed data (test phase: Pearson's coefficient, 76.74 - Root Mean Square error, 1.38; train phase: Pearson's coefficient, 79.53 - Root Mean Square error, 1.29). The small difference between Pearson's coefficient values in different phase, suggests that the model generalizes well and that it is likely to make accurate predictions when it processes new data, as described in the instruction manual of NeuralWorks Predict. Moreover, ANN analysis allows a prediction with an overall accuracy of $83.63 \%$ (Fig. 2) and the selection of the number of lesions, the previous recurrence rate and the response to previous Bacille Calmette-Guérin adjuvant therapy as the most influential variables affecting the output decision in predicting the natural history of pTaG3 urothelial bladder cancer.

\section{Discussion}

In the present study, we developed a simple and rapid method to apply ANN multi-factorial analysis to management of UC of the urinary bladder, by using only common prognostic 
Table II. Univariate and multivariate analysis results of factors affecting recurrence risk in pTaG3 patients.

\begin{tabular}{|c|c|c|c|}
\hline Categories (variables) & $\begin{array}{l}\text { No. of recurrence-free patients/ } \\
\text { No. of total patients }(\%)\end{array}$ & $\begin{array}{l}\text { Univariate analysis (p) } \\
\qquad \mathrm{HR}^{\mathrm{a}}\left(95 \% \mathrm{CI}^{\mathrm{b}}\right)\end{array}$ & $\begin{array}{l}\text { Multivariate analysis (p) } \\
\qquad \operatorname{HR}^{\mathrm{a}}\left(95 \% \mathrm{CI}^{\mathrm{b}}\right)\end{array}$ \\
\hline Age (years) & & 0.828 & 0.721 \\
\hline$<65$ & $32 / 51 \quad(62.7)$ & $1.10(0.98-1.28)$ & $1.06(0.54-1.32)$ \\
\hline$\geq 65$ & $23 / 92(25.0)$ & $0.98(0.67-1.18)$ & $0.84(0.27-1.20)$ \\
\hline Sex & & 0.727 & 0.819 \\
\hline Male & $39 / 116$ (33.6) & $0.96(0.41-1.67)$ & $1.19(0.88-1.81)$ \\
\hline Female & $16 / 27(59.2)$ & $1.28(0.69-1.87)$ & $1.34(0.99-1.67)$ \\
\hline Previous histopathological data & & 0.657 & 0.067 \\
\hline pTaG1 & $14 / 41$ & $0.99(0.39-1.98)$ & $1.02(0.77-2.36)$ \\
\hline pTaG2 & $23 / 74(31.0)$ & $1.33(0.90-1.61)$ & $1.91(0.89-1.97)$ \\
\hline pTaG3 & $18 / 28 \quad(64.2)$ & $1.20(0.60-1.24)$ & $0.97(0.54-1.69)$ \\
\hline Previous recurrence rate & & 0.058 & 0.003 \\
\hline $1 /$ year & $16 / 36(44.4)$ & $1.60(0.46-1.92)$ & $1.01(0.22-1.29)$ \\
\hline $2 /$ year & $27 / 82(32.9)$ & $3.01(2.00-5.27)$ & $3.14(2.19-4.52)$ \\
\hline$\geq 3 /$ year & $12 / 25(48.0)$ & $4.13(3.17-7.14)$ & $4.78(3.12-6.23)$ \\
\hline Previous intravesical treatment & & 0.584 & 0.089 \\
\hline $\mathrm{BCG}^{\mathrm{c}}$ & 28/108 (25.9) & $1.32(1.19-1.67)$ & $1.21(1.03-1.88)$ \\
\hline Mitomycin & $15 / 22(68.1)$ & $1.61(1.54-2.20)$ & $1.23(1.06-1.33)$ \\
\hline None & $12 / 13(92.3)$ & $2.34(1.89-3.12)$ & $3.34(2.32-4.01)$ \\
\hline Diameter of lesions $(\mathrm{cm})$ & & 0.209 & 0.125 \\
\hline $1-1.9$ & $18 / 68$ & $1.14(0.84-1.96)$ & 1.00 \\
\hline $2-2.9$ & $19 / 36(52.7)$ & $1.85(0.67-2.18)$ & $1.87(1.11-2.04)$ \\
\hline 3-3.9 & $12 / 21 \quad(57.1)$ & $2.89(2.12-4.78)$ & $2.68(2.23-3.31)$ \\
\hline $4-4.9$ & $4 / 10(40.0)$ & $3.14(1.96-5.27)$ & $4.01(2.10-6.61)$ \\
\hline$\geq 5$ & $2 / 8 \quad(25.0)$ & $2.79(2.01-4.38)$ & $3.11(2.17-4.89)$ \\
\hline No. of lesions & & 0.123 & 0.008 \\
\hline Single & $38 / 95(40.0)$ & 1.00 & $0.82(0.34-1.11)$ \\
\hline Multiple & $17 / 48 \quad(35.4)$ & $1.98(1.12-3.42)$ & $3.31(2.36-3.85)$ \\
\hline Associated Cis & & 0.843 & 0.981 \\
\hline With Cis & $3 / 8 \quad(37.5)$ & $3.13(2.10-5.22)$ & $2.97(2.00-4.48)$ \\
\hline Without Cis & $52 / 135$ & $1.16(0.78-1.29)$ & $1.33(0.92-1.67)$ \\
\hline Inflammation & & 0.627 & 0.043 \\
\hline With inflammation & $39 / 71 \quad(54.9)$ & $1.70(0.93-2.17)$ & $0.88(0.22-1.26)$ \\
\hline Without inflammation & $16 / 72(22.2)$ & $1.24(0.34-1.61)$ & $2.89(2.10-4.92)$ \\
\hline Adjuvant therapy & & 0.280 & 0.235 \\
\hline $\mathrm{BCG}^{\mathrm{c}}$ & 47/128 (36.7) & $1.10(0.67-1.37)$ & $1.01(0.98-1.03)$ \\
\hline Other & $8 / 15 \quad(53.3)$ & 1.00 & $1.28(0.97-1.44)$ \\
\hline
\end{tabular}

Univariate and multivariate analysis results of factors affecting recurrence risk in all enrolled patients at 12 months follow-up. ${ }^{\text {aHR }}$, hazard ratio; b95\% CI, 95\% confidence interval; 'BCG, Bacille Calmette-Guérin.

factors that are used in every day urological clinical practice. We analyzed the results of the standard prognostic factors, in a group of 143 patients affected by recurrent pTaG3 bladder cancer, by using a long-term follow-up period (mean 108 months). The resulting data at a long-term follow-up were used to identify the most influential variables affecting the output decision in predicting the natural history of pTaG3 urothelial bladder cancer and the ANN creation for predicting the recurrence risk at 12 months follow-up. We have considered data at 12 months of follow-up as indicative of a realistic predictive patient outcome, due to the fact that the higher recurrence rate of pTaG3 patients is during the 


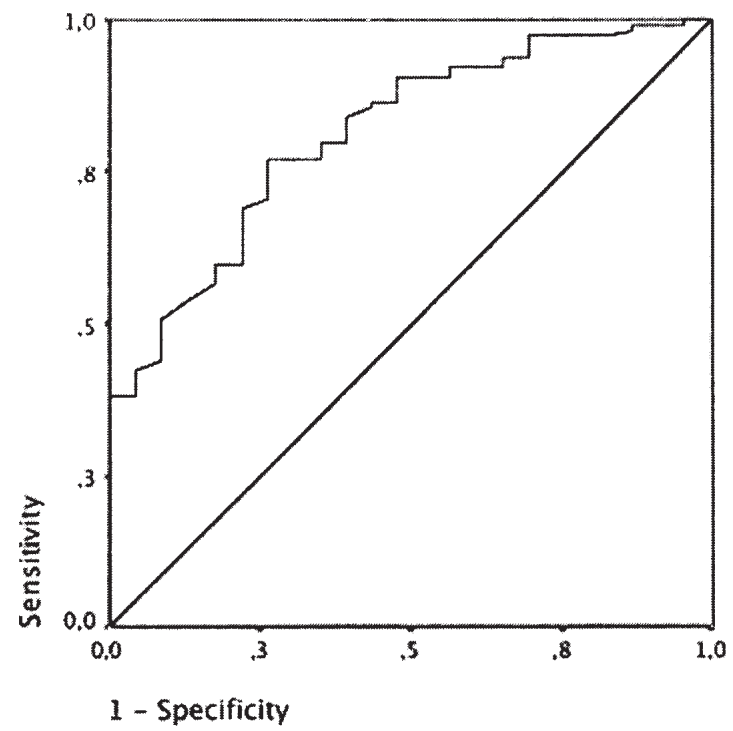

Figure 1. The Receiver Operating Characteristic (ROC) curve used to evaluate the best cut-off value, in the ANN analysis. The area under ROC curve is 0.816 and shows a good test performance. Sensitivity $81.67 \%$, specificity $95.87 \%$, positive predictive value $98.2 \%$, negative predictive value $92.3 \%$.

first year after TUR-BT (18). By using multivariate analysis, we demonstrated that, among all standard clinical and pathological variables, only the previous recurrence rate $(\mathrm{p}=0.003)$, the number of lesions ( single or multiple) $(\mathrm{p}=0.008)$ and the number of recurrences $(\mathrm{p}=0.011)$ are independent factors in predicting recurrence risk. These data are in agreement with other reports $(7,18,20)$. Furthermore, the presence of tumor-associated tissue inflammatory reaction $(\mathrm{p}=0.04)$ can be identified as an independent factor, as reported (19). The ANN led to a recurrence probability prediction in pTaG3 patients with an accuracy of $83.63 \%$,

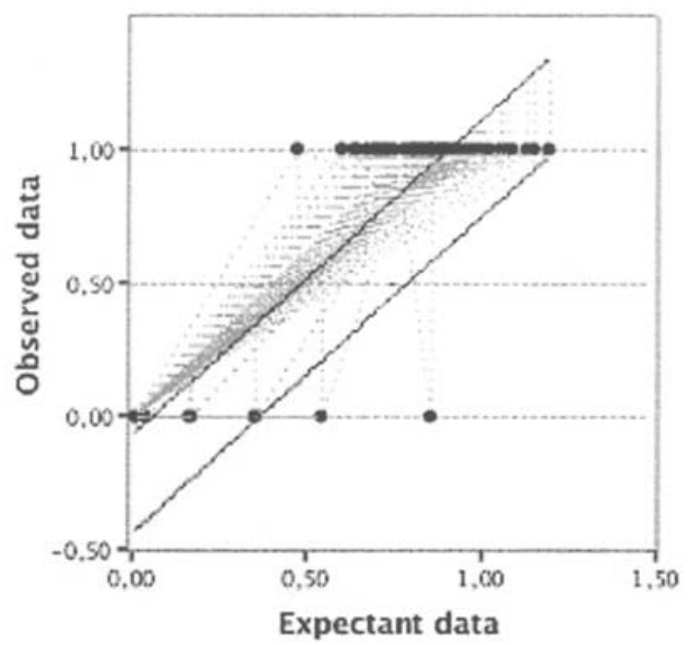

Figure 2. The linear regression with $95.00 \%$ individual prediction interval. R-Square 0.87 .

allowing a more correct and appropriate recurrence risk group stratification. ANN analysis also allowed identification of the number of lesions, the previous recurrence rate and the response to previous Bacille Calmette-Guérin adjuvant therapy as the most influential variables affecting the recurrence risk of pTaG3 urothelial bladder cancer. The number of recurrences and the presence of tumor-associated tissue reaction that have been described as independent prognostic factors in multivariate analysis, have not been identified as independent factors in ANN analysis either. The reason for this difference could be determined by a different impact that each factor has in each statistical or mathematic analysis. The different independent prognostic factor identifications, reported by mathematic analysis (ANN) and by statistical analysis (multivariate analysis), stress the fact that in urothelial bladder tumor natural history and its

Table III. Input variables used in construction of ANN and their contribution in analysis.

\begin{tabular}{|c|c|c|c|}
\hline Variables & Categories & $\begin{array}{c}\Delta^{\mathrm{a}} \\
(\text { mean } \pm \mathrm{SD})^{\mathrm{c}}\end{array}$ & $\begin{array}{l}\text { Multivariat } \\
\text { analysis }^{\mathrm{b}}(\mathrm{p}\end{array}$ \\
\hline Age & $<65$ years, $\geq 65$ years & $11.51 \pm 16.23$ & 0.721 \\
\hline Sex & Male, female & $27.35 \pm 29.26$ & 0.819 \\
\hline Previous histopathological data & pTaG1, pTaG2, pTaG3 & $31.74 \pm 37.91$ & 0.067 \\
\hline Previous recurrence rate & $1 /$ year, $2 /$ year, $3 /$ year or more & $49.01 \pm 49.74$ & 0.003 \\
\hline Response to previous $\mathrm{BCG}^{\mathrm{d}}$ therapy & Yes, no & $48.62 \pm 50.17$ & $n e^{e}$ \\
\hline No. of lesions & Single, multiple & $48.97 \pm 49.89$ & 0.008 \\
\hline Diameter of lesions & $1,2,3,4,5$ or more ${ }^{\mathrm{e}}$ & $28.59 \pm 31.27$ & 0.125 \\
\hline Inflammation & Yes, no & $39.67 \pm 41.26$ & 0.043 \\
\hline Adjuvant therapy after TUR-BT & Yes, no & $31.90 \pm 32.92$ & 0.235 \\
\hline
\end{tabular}

The table shows the input variables used in Artificial Neural Network analysis. ${ }^{a} \Delta$, absolute difference between the original and modified neural net outputs; bultivariate analysis, resulted data from multivariate analysis; ${ }^{\mathrm{c}} \mathrm{SD}$, standard deviation; ${ }^{\mathrm{d}} \mathrm{BCG}$, Bacille Calmette-Guérin; ${ }^{e}$ ne, variable not evaluated in multivariate analysis. 
follow-up decision making we must use different approaches, due to the fact that tumor recurrence or progression is related to a complex network of independent variables. ANN, from this point of view, is able to analyze a non-linear relationship, by using multiple layers of interconnected nodes, and achieve more accurate results (13). The previous histopathological data results do not seem to have a significative prognostic role in the determination of recurrencefree survival prediction in pTaG3 patients. Among all recurrent pTaG3 patients, only this type of patient could benefit from a follow-up with strict observations, as proposed by Sylvester et al (2). From this point of view, the follow-up schedule will not only be planned on the basis of histopathological result but also on that of the complex interconnections between all clinical and pathological variables. For this reason, only the small number of pTaG3 patients who reported the above described characteristics will undergo rigorous follow-up with frequent and invasive instrumental diagnostic methods. The choice to include in this study only patients with pTaG3 at histopathological analysis is due to the fact that, even if they have a low frequency rate (1-4), they have more uncertain clinical and biological behavior that, in half of them, must be treated aggressively as invasive high-grade urothelial carcinoma (23). Moreover, as also highlighted by Sylvester et al, pTaG3 patients are a group, who are subjected to considerable histological misclassification, with a high rate of under-staging and under-treatment (2). The high number of pTaG2 $(51.7 \%)$ at the previous histopathological evaluation could stress this feature. In addition, the use of a multiple factor can also reduce error in follow-up decision making for a population of patients. This study represents encouragement to follow the direction of the use of mathematical applications in everyday clinical practice, for improving the management of cancer patients, even if, as Catto et al underlined, 'the scientific community remains skeptical of ANN' (24).

We demonstrated the feasibility and reliability of ANN applications in everyday urologic practice, reporting a good accuracy in addition to good recurrence predicting performance. The study identified also a single subgroup of pTaG3 patients that seem to be at high risk of recurrence.

\section{Acknowledgements}

Special thanks to Professor John Denton for manuscript language revision, to Dr Jim W.F. Catto of the University of Sheffield for his help in study design and support. Moreover, we are grateful to all Santa Maria Annunziata Hospital Urologic Unit members for their help in patient data collection. This study was supported by a grant from the Department of Urology of the University of Florence.

\section{References}

1. Van der Meijden A, Sylvester R, Collette L, Bono A and Ten Kate F: The role and impact of pathology review on stage and grade assessment of stages Ta and T1 bladder tumors: a combined analysis of 5 European organization for research and treatment of cancer trials. J Urol 164: 1533-1537, 2000.

2. Sylvester RJ, van der Meijden A, Witjes JA, et al: High-grade Ta urothelial carcinoma and carcinoma in situ of the bladder. Urology 66: 90-107, 2005.
3. Heney NM, Ahmed S, Flanagan MJ, et al: Superficial bladder cancer: progression and recurrence. J Urol 130: 1083-1086, 1983.

4. Holmäng S, Andius $\mathrm{P}$, Hedelin $\mathrm{H}$, Wester K, Busch C and Johansson SL: Stage progression in Ta papillary urothelial tumors: relationship to grade, immunohistochemical expression of tumor markers, mitotic frequency and DNA ploidy. J Urol 165: 1124-1130, 2001.

5. Herr HW: Tumor progression and survival of patients with high grade, non-invasive papillary (TaG3) bladder tumors: 15-year outcome. J Urol 163: 60-62, 2000.

6. Fujimoto $\mathrm{K}$, Chihara $\mathrm{Y}$, Kondo $\mathrm{H}$ and Hirao $\mathrm{Y}$ : Initial conservative treatment for grade $3 \mathrm{Ta}-1$ superficial bladder cancer. Hinyokika Kiyo 52: 433-438, 2006.

7. Millan-Rodriguez F, Chechile-Toniolo G, Salvador-Bayarri J, Palou J and Vicente-Rodriguez J: Multivariate analysis of the prognostic factors of primary superficial bladder cancer. J Urol 163: 73-78, 2000.

8. Stein JP, Grossefeld GD, Ginsberg DA, et al: Prognostic markers in bladder cancer: a contemporary review of the literature. $J$ Urol 160: 645-659, 1998.

9. Habuchi T, Marberger M, Droller MJ, et al: Prognostic markers for bladder cancer: International Consensus Panel on bladder tumor markers. Urology 66 (Suppl. 1): S64-S74, 2005.

10. Müller B, Reinhardt J and Strickland MT (eds): Neural network introduced. In: Neural Networks: An Introduction. Chapt. 2. 2nd edition. Springer-Verlag, New York, pp13-23, 1995.

11. Sargent DJ: Comparison of artificial networks with other statistical approaches. Cancer 91: 1636-1642, 2001.

12. Qureshi KN, Naguib RNG, Hamdy FC, Neal DE and Mellon JK: Neural network analysis of clinicopathological and molecular markers in bladder cancer. J Urol 163: 630-633, 2000.

13. Wei JT, Zhang Z, Barnhill SD, Madyastha KR, Zhang H and Oesterling JE: Understanding artificial neural networks and exploring their potential applications for the practicing urologist. Urology 52: 161-172, 1998.

14. Mattfeldt HA, Kestler R, Hautmann R and Gottfried HW: Prediction of prostatic cancer progression after radical prostatectomy using artificial neural networks: a feasibility study. BJU Int 84: 316-323, 1999.

15. Catto JW, Linkens DA, Abbod MF, et al: Artificial intelligence in predicting bladder cancer outcome: a comparison of neurofuzzy modeling and artificial neural networks. Clin Cancer Res 9: 4172-4177, 2003.

16. Abbod MF, Linkens DA, Catto JW and Hamdy FC: Comparative study of intelligent models for the prediction of bladder cancer progression. Oncol Rep 15: 1019-1022, 2006.

17. Cai T, Conti G, Lorenzini $\mathrm{M}$ and Bartoletti R: Artificial intelligences in urological practice: the key to success? Ann Oncol 18: 604-605, 2007.

18. Oosterlinck W, Lobel B, Jakse G, Malmström P, Stöckle M and Sternberg C: (The EAU Working Group on Oncological Urology): Guidelines on bladder cancer. Eur Urol 41: 105-112, 2002.

19. Cai T, Nesi G, Boddi V, Mazzoli S, Dal Canto M and Bartoletti R: Prognostic role of the tumor-associated tissue inflammatory reaction in transitional bladder cell carcinoma. Oncol Rep 16: 329-334, 2006.

20. Kurth KH, Denis L, Bouffioux C, Sylvester R, Debruyne FM and Pavone-Macaluso M: Factors affecting recurrence and progression in superficial bladder tumours. Eur J Cancer 31A: 1840-1846, 1995

21. Solsona E, Iborra I, Dumont R, Rubio-Briones J, Casanova JL, and Almenar S: The 3-month clinical response to intravesical therapy as a predictive factor for progression in patients with high-risk superficial bladder cancer. J Urol 164: 685-689, 2000.

22. Herr HW and Dalbagni G: Defining bacillus Calmette-Guerin refractory superficial bladder tumors. J Urol 169: 1706-1708, 2003.

23. Lebret T, Bohin D, Kassardjian Z, et al: Recurrence, progression and success in stage Ta grade 3 bladder tumors treated with lowdose bacillus Calmette-Guerin instillations. J Urol 163: 63-67, 2000.

24. Catto JW, Abbod MF, Linkens DA and Hamdy FC: Neurofuzzy modelling: an accurate and interpretable method for predicting bladder cancer progression. J Urol 175: 474-479, 2006. 